\title{
Mitochondrial Uncoupling Proteins: A Potential Target for Disease-Modulation in Cardiovascular Disorders
}

\author{
Zakaria A Almsherqi* \\ Department of Physiology, Singapore
}

*Corresponding author: Zakaria A Almsherqi, Yong Loo Lin School of Medicine, National University of Singapore, Singapore.

Received Date: May 25, 2019

Published Date: May 28, 2019

\begin{abstract}
Mitochondria provide cellular energy through oxidative phosphorylation, but as an integral part of this process, superoxides and other reactive oxygen species (ROS) are also produced. When the balance between the production of free radicals and antioxidant capacity of the cardiac cells altered, oxidative stress is induced. Oxidative stress has been linked to the development of ischemic heart disease, atherosclerosis, congestive heart failure, ischemic-reperfusion injury, and vascular endothelial dysfunction. Although several clinical trials over the past decades employed different strategies of antioxidants supplementation, the results were generally negative in the setting of chronic preventative therapy. Less attention has been paid to the modulation of ROS production, despite the fact that prevention, rather than cure, would appear to be the logic approach to attenuate the oxidative damage. There is increasing evidence that endogenous myocardial uncoupling proteins (UCPs) can play a vital role in regulating ROS generation to protect against various pathogenic stresses. Their expression, which can be induced, may well be a potential therapeutic target for various drugs to alleviate the harmful effects of pathogenic processes and hence modify the progression of cardiovascular diseases (CVDs).

Myocardial function depends on the energy that it is able to synthesize and transfer in the form of energy-rich energy for phosphate bonds to fuel excitation-contraction coupling. More than $90 \%$ of cardiac cells energy is produced in the mitochondria from oxidative phosphorylation activity [1]. Oxidative phosphorylation is the process by which energy from fuel oxidation is converted to the high-energy phosphate bonds of adenosine triphosphate (ATP). The chemiosmotic hypothesis suggests that the ATP synthesis is provided by the electrochemical gradient across the inner mitochondrial membrane. This electrochemical gradient is maintained by constituents of the electron transport chain (ETC), which acts to pump protons from the mitochondrial matrix to the intermembrane space of the mitochondria as they accept and donate electrons in a prescribed manner Figure. This process of electron transport and oxidative metabolism in cardiac cells are accompanied by the reduction of oxygen to superoxide and other ROS which considered as a by-product of mitochondrial respiration.
\end{abstract}

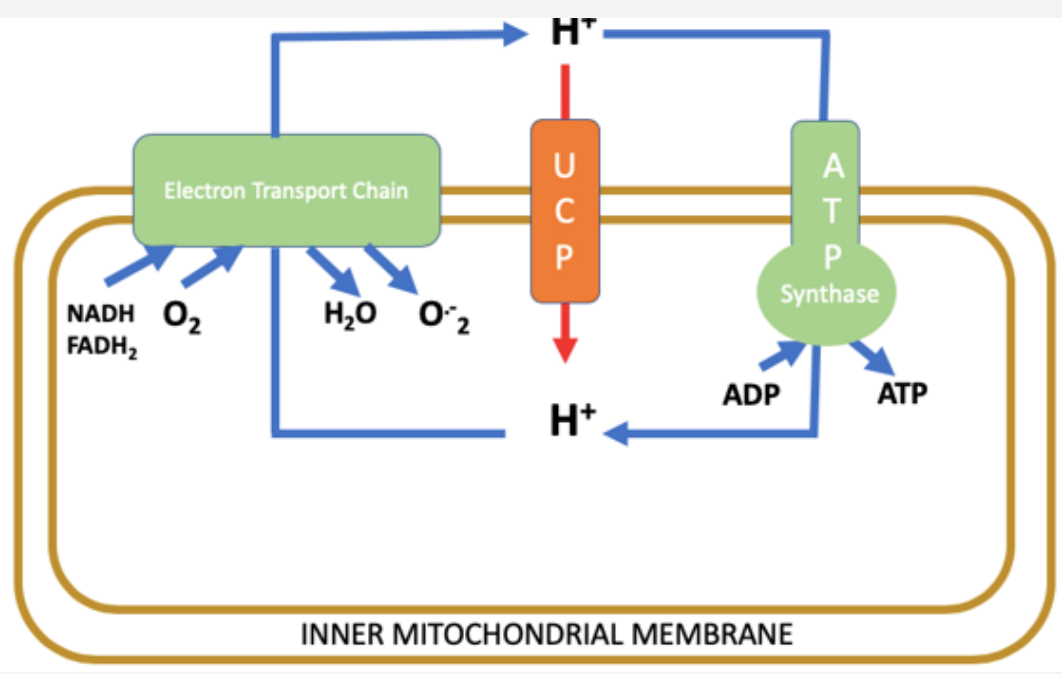

Figure 1: The chemiosmotic proton cycle across the inner mitochondrial membrane. The coupling cycle, consisting of substrate oxidation (NADH, FADH2) and the enzymes of ATP production (ATP synthase), results in coupled oxidative phosphorylation and superoxide (O-2) generation as a by-product. The uncoupling cycle, consisting of substrate oxidation and proton conductance pathway through the uncoupling proteins (UCPs), results in uncoupled respiration and low levels of superoxide generated. 


\section{Redox Status of Cardiac Cells: The "Uncoupling" Link}

An appreciation of the ETC and its role in oxidative phosphorylation is essential in the understanding of the clinical significance of UCPs. In the uncoupling process, as the name suggests, the electrochemical gradient is restored independently of the activity of ATP synthase [2]. A considerable level of basal proton leak, also known as global proton leak, occurs across the inner mitochondrial membrane all the time [3]. While most of this leak is attributed to the action of uncoupling proteins Figure, the permeability of the mitochondrial membrane due to the proteins embedded in its lipid bilayer also contributes to the membrane's leakiness. Approximately 20\% of the body's resting metabolic rate is used to maintain the electrochemical gradient that is dissipated by this basal proton leak [4]. This significant mitochondrial proton leak should serve an important function in view of the high energetic cost utilized to maintain it.

There is increasing evidences show that UCPs, by mitochondrial uncoupling, protect the heart by reducing ROS generated by the mitochondria. As a result, cardiomyocyte could be protected from stress-induced apoptosis [5]. UCP3, in particular, has been associated with cellular fatty acid metabolism, with its distribution being most pronounced in muscles with high-fat oxidative capacity (such as cardiac cells) [6]. In physiological or pathological conditions where plasma fatty acid levels increase, UCP3 is upregulated. On the contrary, a decrease in plasma fatty acid levels causes downregulation of UCP3. These seem to support the hypothesis that UCP3 plays an important role in exporting fatty acids that cannot be oxidized from the mitochondrial matrix, thereby inhibiting the accumulation of fatty acids inside the matrix. In this way, UCP3 provides protection from lipid-induced mitochondrial damage and mitochondria-dependent apoptosis [6].

\section{UCP Regulation in Cardiac Cell}

UCP expression is regulated in response to externals stressors by a host of transcription factors. In particular, peroxisome proliferator-activated receptor alpha (PPAR $\alpha$ ) and peroxisome proliferator-activated receptor gamma coactivator 1 alpha (PGC1- $\alpha$ ). Both transcription factors play an essential role in the response to external environmental stress, such as fasting and physical stress. Furthermore, activation and/or expression of UCPs is also closely monitored by intra and extracellular factors. Regulation may be achieved by enzymes such as AMP-activated protein kinase (AMPK), proteins such as sterol-responsive elementbinding protein (SREBP) or nuclear transcription factors such as PPAR.

\section{Modulation of oxidative stress as a potential therapeutic target}

Overexpression of UCPs has a beneficial effect on cardiac energetics regulation, mitochondrial ROS production, calcium handling and cardiomyocyte apoptosis [7]. Increased UCP expression could also protect the endothelial cells from the cytotoxic effect of lysophosphatidylcholine associated with vascular diseases [8]. However, it may have opposite effects on other CVDs. The associated decrease in ATP synthesis with overexpression of UCP may have deleterious consequences on cardiac function and may worsen the clinical outcomes. Thus, modulation of oxidative stress through UCPs regulation in CVD as a therapeutic option should be considered carefully.

The observed downregulation of UCP in heart failure and subsequent inability of the cell to combat the oxidative burden caused by the failing heart has led to a potential therapeutic role of UCP to compact the pathogenesis of the disease. Pathogenesis of heart failure has been characterized by an increase in ROS production and ROS-mediated damage. UCPs are known to prevent ROS accumulation and hence decrease the oxidative burden by limiting ROS production. Furthermore, UCPs may be involved in the detoxification of exogenously-produced ROS. In the setting of heart failure, there is an increase in the concentration of circulating free fatty acids. This is positively correlated with an increase in cardiac mitochondrial UCPs. The metabolic effects of UCPs are cardioprotective in nature and are in fact an adaptive response to the rise in lipid concentration in the mitochondria [9]. Therefore, upregulating UCP expression in heart failure could be of therapeutic benefit.

One of the key regulators of UCP expression in the heart is PPAR [10]. Hence, to upregulate UCP expression, agonists of PPAR could be used. Currently few ongoing studies use PPAR agonists to test their effects on obesity control, diabetes treatment as well as CVDs. AMPK has been implicated to have a protective role (anti-apoptotic) as it enhances the survival of cardiomyocytes in response to ischemia and reperfusion. Loss of AMPK activity results in an inability to increase glucose uptake and glycolysis by the cardiomyocytes [11]. Taken together, as a result of AMPK role in mediating the survival of cardiomyocytes during ischemia, is it also a potential target for augmenting the expression of UCP in heart failure.

Another translational approach would be the development of drugs that would induce UCP expression and hence slow the progression of atherosclerosis and endothelial dysfunction. The effects of sitagliptin, a fibrate vegetable extracts, have been shown to increase the expression of UCP2 with an associated improvement in mitochondrial biogenesis and function [12]. As such, the development of a therapeutic agent that modulates oxidative stress would be of significant impact on vascular diseases.

In conclusion, despite considerable medical interest, the molecular mechanisms that regulate ROS formation within the mitochondrion remain poorly investigated. However, increasing evidence indicates that myocardial UCPs may well play a crucial role in cellular survival when they are under stress. Myocardial UCPs may be a potential therapeutic target for the treatment of cardiovascular disorders in the near future.

\section{Acknowledgement}

None.

\section{Conflict of Interest}

No conflict of interest. 


\section{References}

1. Mootha VK, AE Arai, RS Balaban (1997) Maximum oxidative phosphorylation capacity of the mammalian heart. American Journal of Physiology-Heart and Circulatory Physiology 272(2): H769-H775.

2. Brand MD, TC Esteves (2005) Physiological functions of the mitochondrial uncoupling proteins UCP2 and UCP3. Cell metabolism 2(2): 85-93.

3. Nicholls DG (1974) The influence of respiration and ATP hydrolysis on the proton-electrochemical gradient across the inner membrane of ratliver mitochondria as determined by ion distribution. European Journal of Biochemistry 50(1): 305-315.

4. Rolfe DF, Newman JM, Buckingham JA, Clark MG, Brand MD, et al. (1999) Contribution of mitochondrial proton leak to respiration rate in working skeletal muscle and liver and to SMR. American Journal of PhysiologyCell Physiology 276(3): C692-C699.

5. Akhmedov AT, Rybin V, Marín-García J (2015) Mitochondrial oxidative metabolism and uncoupling proteins in the failing heart. Heart failure reviews 20(2): 227-249.

6. Schrauwen P, Hesselink MK (2004) The role of uncoupling protein 3 in fatty acid metabolism: protection against lipotoxicity? Proceedings of the Nutrition Society 63(2): 287-292.
7. Sack MN (2006) Mitochondrial depolarization and the role of uncoupling proteins in ischemia tolerance. Cardiovascular research 72(2): 210-219.

8. Chang MC, Lee JJ, Chen YJ, Lin SI, Lin LD, et al. (2017) Lysophosphatidylcholine induces cytotoxicity/apoptosis and IL-8 production of human endothelial cells: Related mechanisms. Oncotarget 8(63): 106177-106189.

9. Hesselink MK, P Schrauwen (2005) Uncoupling proteins in the failing human heart: friend or foe? The Lancet 365(9457): 385-386.

10. Young ME, Patil S, Ying J, Depre C, Ahuja HS, et al. (2001) Uncoupling protein 3 transcription is regulated by peroxisome proliferator-activated receptor $\alpha$ in the adult rodent heart. The FASEB Journal 15(3): 833-845.

11. Russell RR, Li J, Coven DL, Pypaert M, Zechner C, et al. (2004) AMPactivated protein kinase mediates ischemic glucose uptake and prevents postischemic cardiac dysfunction, apoptosis, and injury. The Journal of clinical investigation 114(4): 495-503.

12. Pierelli G, Stanzione R, Forte M, Migliarino S, Perelli M, et al. (2017) Uncoupling Protein 2: A Key Player and a Potential Therapeutic Target in Vascular Diseases. Oxidative medicine and cellular longevity 2017:7348372. 